\title{
Frequent consumption of vegetables predicts lower risk of depression in older Taiwanese - results of a prospective population-based study
}

\author{
Alan C Tsai ${ }^{1,2, *}$, Tsui-Lan Chang $^{3}$ and Shu-Hwang $\mathrm{Chi}^{1}$ \\ 'Department of Healthcare Administration, Asia University, 500 Liufeng Road, Wufeng, Taichung 41354, \\ Taiwan, Republic of China: ${ }^{2}$ Department of Health Services Management, School of Public Health, \\ China Medical University, Taichung, Taiwan, Republic of China: ${ }^{3}$ Nursing Department, Hsin Yung Ho Hospital, \\ Taoyuan, Taiwan, Republic of China
}

Submitted 30 May 2011: Accepted 11 October 2011: First published online 16 December 2011

\begin{abstract}
Objective: The study evaluated the association between consumption frequencies of the major food categories and the risk of new depression four years later in older Taiwanese.

Design: A prospective cohort study with multistage random sampling. Logistic regression analysis evaluated the significance of the longitudinal associations of intake frequencies of the major food categories with future ( 4 years later) risk of new depression, controlled for possible confounding factors with or without adjustment for cognitive status.

Setting: Population-based free-living elderly.

Subjects: Men and women $(n$ 1609) $\geq 65$ years of age.

Results: In a regression model that controlled for demographic, socio-economic, lifestyle and disease/health-related variables but not cognitive status, both fruits $(\mathrm{OR}=0 \cdot 66,95 \% \mathrm{CI} 0 \cdot 45,0 \cdot 98, P=0 \cdot 038)$ and vegetables $(\mathrm{OR}=0 \cdot 38,95 \% \mathrm{CI}$ $0 \cdot 17,0 \cdot 86, P=0 \cdot 021)$ were protective against depressive symptoms 4 years later. However, when the same regression model was also adjusted for cognitive status, only vegetables $(\mathrm{OR}=0 \cdot 40,95 \% \mathrm{CI} 0 \cdot 17,0 \cdot 95, P=0 \cdot 039)$ were protective against depressive symptoms. Higher consumption of eggs was close to being significant in both regression models $(P=0.087$ and 0.069 , respectively). Other food categories including meat/poultry, fish, seafood, dairy, legumes, grains and tea showed no significant associations.

Conclusions: Results suggest that although confounding factors cannot be totally ruled out, more frequent consumption of vegetables seems to be protective against depressive symptoms in the elderly. Further studies are needed to elucidate the causal role and the mechanism of the association.
\end{abstract}

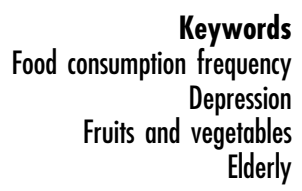

Depression is a common mental health condition and its incidence increases exponentially with age ${ }^{(1)}$. A growing body of epidemiological evidence including both crosssectional and longitudinal studies suggests a relationship between diet/nutrition and mental health. Considerable research effort has been devoted to analysing the possible association of dietary pattern with depressive symptoms ${ }^{(2-5)}$. In cross-sectional studies, poor habitual diet quality has been observed to be associated with high prevalence of mental disorders in Australian women ${ }^{(6)}$; an unhealthy food consumption pattern was found to be associated with depressive symptoms in female but not male college students in three European countries ${ }^{(4)}$; and a healthy dietary pattern was found to be associated with lower prevalence of depressive status in male and female Japanese municipal employees ${ }^{(7)}$.

A prospective study conducted by Akbaraly et al. ${ }^{(8)}$ showed that a processed foods dietary pattern high in sweet deserts, fried food, processed meat, refined grains and high-fat dairy products was a risk factor for depression 5 years later as measured by the CES-D (Center for Epidemiologic Studies Depression Rating Scale), whereas a whole foods pattern high in vegetables, fruits and fish was protective in middle-aged (35-55 years old) participants.

The elderly are at higher risk of both depression and nutritional deficiency ${ }^{(1)}$. Wang $^{(9)}$ showed that $57 \%$ of rural elderly Taiwanese had depressive symptoms. Many elderly also have depressed appetite, ageing-related 
anorexia, poor dental status and functional or cognitive impairments. They are more inclined to eat a simple and nutritionally inadequate diet, and therefore have increased risk of nutritional deficiency. Low dietary and blood levels of certain nutrients such as vitamin $\mathrm{B}_{6}$, vitamin $\mathrm{B}_{12}$, folic acid and $n-3$ fatty acids might be associated with increased risk of depressive symptoms ${ }^{(2,10)}$. A recent study observed a longitudinal association of dietary total intake of vitamins $\mathrm{B}_{6}, \mathrm{~B}_{12}$ and folate with depressive symptoms among older adults over time ${ }^{(11)}$. Thus, it is hypothesized that what we eat may impact our psychological health by affecting nutrient levels in the blood. In addition, the psychological well-being of older adults could also be impacted by unfavourable socio-economic, environmental, lifestyle and health-related factors. Thus, the present study aimed to examine the longitudinal association of the frequencies of consumption of the major food categories with the risk of new depression in elderly Taiwanese.

\section{Methods}

\section{Source of data and participants}

Data used for this analysis were from the 1999 and 2003 'Survey of Health and Living Status of the Elderly in Taiwan' (SHLSET), a prospective study undertaken by the Bureau of Health Promotion of Taiwan. The survey was initiated in 1989 and employed a multistage random sampling process to draw a national random sample of 4412 Taiwanese aged 60 years or older. A second sample of 2462 Taiwanese, 50-66 years old, was selected with the same process and added to the cohort in 1996 in order to maintain and extend the age range of the cohort. The combined cohort was interviewed every 3-4 years (1989, 1992, 1996, 1999, 2003 and 2007). The 1999 survey contained more detailed information on participants' dietary pattern and nutritional status and thus was chosen as baseline of the present analysis. The 2003 survey served as endpoint. The completion rates were $90 \cdot 3 \%$ for the 1999 survey and $91.6 \%$ for the 2003 survey. Of 4440 participants who completed the 1999 survey, 2890 met the age requirement ( $\geq 65$ years) of the present study. After excluding 905 who had depressive symptoms (CES-D score $\geq 10$ ) at baseline and 376 who failed to complete the 2003 survey or died, data from the remaining 1609 participants were analysed in the present study. The design and questionnaire of the survey is available at a government website ${ }^{(12)}$ and described in a recent publication $^{(13)}$. The protocol of SHLSET was reviewed and approved by government-appointed representatives and the study was conducted according to ethical standards set forth in the Helsinki Declaration. All participants gave informed consent and their anonymity was preserved at all times.

\section{Study variables}

In each survey, trained interviewers conducted inhome in-person interviews to elicit sociodemographic, anthropometric, health and health-care-related information from each participant. Among other questions, the questionnaire inquired about the consumption frequencies of the major food categories (including meat and poultry, fish, seafood, eggs, fruits, vegetables and infused camellia tea) during the 1999 interview by a specific question: 'How many times per week do you consume each of the following foods?' The frequencies provided for each food category were 'every day or nearly every day', '3-5 times/week', '1-2 times/week', 'less than 1 time/week' and 'I do not eat such foods'. A separate question asked about the number of bowls of rice or noodles consumed each day. The food consumption questionnaire was pretested and validated with a $14 \mathrm{~d}$ food diary (analysis with Wilcoxon's signed-rank test showed good agreement between the two methods, all $P<0 \cdot 05$ ) prior to the survey (AC Tsai and MC Chang, unpublished results). In the present study, a frequency of $\geq 3$ times/week was arbitrarily chosen as the cut-off point to designate more frequent consumption of a food category. A consumption of three bowls of rice/noodles per day was also the cut-off point for grain intake. Data gathered in the survey were found to have acceptable agreement with that obtained clinically ${ }^{(14,15)}$.

The psychological health status was the dependent variable of the present study. The risk of having a depression was evaluated with CES-D10 in 1999 and in 2003. The ten-item CES-D (CES-D10) was shown to preserve the same sensitivity and specificity as the twenty-item scale and it performed well in cross-cultural and older population studies, including the elderly Chinese. The scale has a maximum score of 30 . A score of 10 or higher was considered having a risk of depression. The cut-off point was shown to have good sensitivity $(0 \cdot 85)$ and specificity $(0 \cdot 80)$ in Chinese elderly ${ }^{(16,17)}$. Cognitive status was rated with the Short Portable Mental Status Questionnaire (SPMSQ) ${ }^{(18)}$. Functional dependency was measured with the Instrumental Activities of Daily Living (IADL) scale. The IADL scale has two scores, one measures if the person has difficulty in carrying out each of six items and the other measures the degree of difficulty in performing the tasks. Participants were asked whether they had difficulty shopping, handling finances, transporting, housekeeping, using telephone or doing some heavy housework. An item that one 'cannot do at all independently' is considered 'dependent' for that item. Participants also rated the level of difficulty for performing each of the six items on a scale from 0 to 3 points $(0=$ no difficulty, 1 = some difficulty, 2 = much difficulty, 3 = cannot do). Individual scores were summed across each of the six items to yield a total score from 0 to 18 , with higher scores reflecting a higher level of ADL difficulty ${ }^{(19,20)}$.

\section{Statistical analysis}

Univariate analysis was performed to evaluate the association of the consumption frequency of each item with current risk of depression (data not shown). Logistic regression analysis was performed to evaluate the association 
of baseline food consumption frequencies with the risk of new depression four years later in elderly who were without depressive symptoms (CES-D $\leq 9)$ at baseline. Because cognitive status has been shown to impact the scoring on the CES-D scale in the elderly, we analysed associations with and without this variable (SPMSQ) in separate regression models. Both regression models were adjusted for demographic status, lifestyle, socio-economic and health-related variables at endpoint. All statistical analyses were performed with the SPSS statistical software package version 15.0 (SPPSS Inc., Chicago, IL, USA). All values were weighting-adjusted according to study design. Statistical significance was evaluated at $\alpha=0 \cdot 05$.

\section{Results}

The study included slightly more men (54.7\%) than women, which reflected the composition of the generation. Table 1 shows the characteristics of the participants according to depression status (CES-D score $\geq 10 v$. $\leq 9$ ) at baseline. Compared with those who had less risk of depression, those who had greater risk of depression were more likely to be female, older, have fewer years of formal education, be less satisfied with their economic status, do less physical activity and have poorer selfviewed health.

Univariate analysis showed that more frequent consumption of all food categories examined was significantly $(P<0.05)$ associated with lower risk of depression (data not shown). Table 2 shows the results of logistic regression analyses. In a regression model without adjusting for cognitive status (SPMSQ score), frequent consumption of fruits and vegetables at baseline was associated with reduced risks of new depression four years later. However, only the consumption of vegetables was significantly associated with reduced risk of depression when the regression model was adjusted for cognitive status (SPMSQ score). Both models also controlled for age, gender and years of formal education at baseline, and economic status, living setting, smoking status, alcohol drinking, betel-nut chewing, functional status, physical exercise and the presence of co-morbidities (hypertension, diabetes, heart disease, cancer, stroke, chronic kidney disease, gout, joint pain/arthritis, gallbladder/liver disease, hip fracture and lower-back pain) at endpoint.

\section{Discussion}

Results of the present study suggest that vegetables are protective against depressive symptoms over time in older adults. In a regression model that controlled for age, gender, years of formal education, satisfaction with economic status, living setting, smoking status, alcohol drinking, betel-nut chewing, functional status (IADL impairments),
Table 1 Characteristics of participants at baseline: free-living elderly men and women $(n 1609) \geq 65$ years of age, Taiwan, 1999 and 2003

\begin{tabular}{|c|c|}
\hline Item & $\%^{*}$ \\
\hline \multicolumn{2}{|l|}{ Sex } \\
\hline Men & $57 \cdot 6$ \\
\hline Women & $42 \cdot 4$ \\
\hline \multicolumn{2}{|l|}{ Age (years) } \\
\hline $65-74$ & $64 \cdot 9$ \\
\hline $75-84$ & $32 \cdot 4$ \\
\hline$\geq 85$ & $2 \cdot 7$ \\
\hline \multicolumn{2}{|c|}{ Formal education (years) } \\
\hline$\leq 6$ & $74 \cdot 8$ \\
\hline $7-12$ & $18 \cdot 5$ \\
\hline$>12$ & $6 \cdot 7$ \\
\hline \multicolumn{2}{|c|}{ Satisfaction with economic status } \\
\hline Satisfied & $44 \cdot 3$ \\
\hline Fair & $42 \cdot 9$ \\
\hline Not satisfied & $12 \cdot 7$ \\
\hline \multicolumn{2}{|c|}{ Whether lives alone } \\
\hline Yes & $9 \cdot 3$ \\
\hline No & $90 \cdot 7$ \\
\hline \multicolumn{2}{|l|}{ Current smoker } \\
\hline Yes & $23 \cdot 4$ \\
\hline No & $76 \cdot 6$ \\
\hline \multicolumn{2}{|c|}{ Alcohol drinking (times/week) } \\
\hline$<1$ & $85 \cdot 5$ \\
\hline$\geq 1$ & $14 \cdot 5$ \\
\hline \multicolumn{2}{|c|}{ Betel-nut chewing } \\
\hline Yes & $4 \cdot 7$ \\
\hline No & $95 \cdot 3$ \\
\hline \multicolumn{2}{|c|}{ Routine physical activity (d/week) } \\
\hline 0 & $29 \cdot 2$ \\
\hline $1-2$ & $5 \cdot 3$ \\
\hline$\geq 3$ & $65 \cdot 4$ \\
\hline \multicolumn{2}{|c|}{ Self-perceived health status } \\
\hline Good & $38 \cdot 8$ \\
\hline Fair & $38 \cdot 0$ \\
\hline Poor & $23 \cdot 2$ \\
\hline
\end{tabular}

*Of 4440 participants who completed the 1999 survey, 2890 met the age requirement ( $\geq 65$ years) of the present study. Among them, 905 who had depressive symptoms (Center for Epidemiologic Studies Depression Rating Scale, score $\geq 10$ ) at baseline and 376 who failed to complete the 2003 survey or died, were excluded. The remaining 1609 participants were included in the present analysis.

physical exercise, hypertension, diabetes, heart disease, cancer, stroke, chronic kidney disease, gout, joint pain/ arthritis, gallbladder/liver disease, hip fracture, lower-back pain and cognitive status (SPMSQ score) at endpoint, more frequent consumption of vegetables was significantly associated with a reduced risk of depression four years later in older adults. Fruit consumption was significant only if the regression model was not adjusted for cognitive status.

In several cross-sectional studies, consumption of vegetables and/or fruits has been shown to be associated with lower risks of depression. Avila-Funes et al. ${ }^{(21)}$ found that intakes of fruits and vegetables (also meat and poultry, fish and dairy) were significantly lower in older Mexican who had depressive symptoms; Woo et al. ${ }^{(3)}$ observed an inverse association of fibre and vegetable intakes with depression in community-living elderly Chinese in Hong Kong; Verger et al. ${ }^{(22)}$ observed that low consumption of fruits and vegetable was associated with 
Table 2 Logistic regression analyses of the association between consumption frequencies of major food categories at baseline and risk of new depression (CES-D $\geq 10$ ) within 4 years, controlled for demographic, socio-economic, lifestyle and health-related variables at endpoint: free-living elderly men and women $(n 1609) \geq 65$ years of age, Taiwan, 1999 and 2003

\begin{tabular}{|c|c|c|c|c|c|c|c|c|}
\hline \multirow[b]{2}{*}{ Variable* $^{\star}$} & \multirow[b]{2}{*}{$\%$ of total } & \multirow[b]{2}{*}{$\%$ Dep } & \multicolumn{3}{|c|}{ Model without SPMSQ } & \multicolumn{3}{|c|}{ Model with SPMSQ } \\
\hline & & & OR & $95 \% \mathrm{Cl}$ & $P$ & OR & $95 \% \mathrm{Cl}$ & $P$ \\
\hline \multicolumn{9}{|c|}{ Meat and poultry (times/week) } \\
\hline$<3$ & $42 \cdot 4$ & $21 \cdot 1$ & $1 \cdot 00$ & & & $1 \cdot 00$ & & \\
\hline$\geq 3$ & $57 \cdot 6$ & $19 \cdot 8$ & $1 \cdot 18$ & $0 \cdot 83,1 \cdot 67$ & $0 \cdot 361$ & $1 \cdot 31$ & $0.90,1.91$ & $0 \cdot 158$ \\
\hline \multicolumn{9}{|c|}{ Dairy (times/week) } \\
\hline$<3$ & $48 \cdot 1$ & $21 \cdot 7$ & $1 \cdot 00$ & & & $1 \cdot 00$ & & \\
\hline$\geq 3$ & $51 \cdot 9$ & $19 \cdot 1$ & 0.95 & $0 \cdot 68,1 \cdot 32$ & $0 \cdot 756$ & $0 \cdot 87$ & $0.61,1 \cdot 24$ & 0.426 \\
\hline \multicolumn{9}{|c|}{ Eggs (times/week) } \\
\hline$<3$ & $55 \cdot 8$ & $23 \cdot 5$ & $1 \cdot 00$ & & & $1 \cdot 00$ & & \\
\hline$\geq 3$ & $44 \cdot 1$ & $16 \cdot 4$ & 0.75 & $0.53,1.04$ & 0.087 & 0.73 & $0.50,1.03$ & 0.069 \\
\hline \multicolumn{9}{|c|}{ Legumes (times/week) } \\
\hline$<3$ & $55 \cdot 2$ & $21 \cdot 6$ & $1 \cdot 00$ & & & 1.00 & & \\
\hline$\geq 3$ & $44 \cdot 8$ & $18 \cdot 8$ & $1 \cdot 10$ & $0 \cdot 79,1 \cdot 54$ & 0.561 & $1 \cdot 08$ & $0 \cdot 76,1 \cdot 55$ & 0.655 \\
\hline \multicolumn{9}{|c|}{ Seafood (times/week) } \\
\hline$<3$ & $88 \cdot 1$ & $21 \cdot 6$ & $1 \cdot 00$ & & & $1 \cdot 00$ & & \\
\hline$\geq 3$ & $11 \cdot 9$ & $13 \cdot 8$ & $0 \cdot 89$ & $0.51,1.56$ & 0.688 & 0.92 & $0.51,1.65$ & 0.773 \\
\hline \multicolumn{9}{|c|}{ Fish (times/week) } \\
\hline$<3$ & $30 \cdot 8$ & $22 \cdot 2$ & $1 \cdot 00$ & & & $1 \cdot 00$ & & \\
\hline$\geq 3$ & $69 \cdot 2$ & $19 \cdot 5$ & 0.92 & $0 \cdot 64,1 \cdot 31$ & 0.633 & 0.91 & $0 \cdot 62,1 \cdot 14$ & $0 \cdot 622$ \\
\hline \multicolumn{9}{|c|}{ Fruits (times/week) } \\
\hline$<3$ & $20 \cdot 6$ & $29 \cdot 3$ & $1 \cdot 00$ & & & $1 \cdot 00$ & & \\
\hline$\geq 3$ & $79 \cdot 4$ & $18 \cdot 0$ & $0 \cdot 66$ & $0.45,0.98$ & 0.038 & 0.77 & $0 \cdot 50,1 \cdot 17$ & $0 \cdot 215$ \\
\hline \multicolumn{9}{|c|}{ Vegetables (times/week) } \\
\hline$<3$ & $2 \cdot 8$ & $37 \cdot 8$ & 1.00 & & & $1 \cdot 00$ & & \\
\hline$\geq 3$ & $97 \cdot 2$ & $19 \cdot 8$ & 0.38 & $0 \cdot 17,0 \cdot 86$ & 0.021 & $0 \cdot 40$ & $0.17,0.95$ & 0.039 \\
\hline \multicolumn{9}{|c|}{ Cereal (bowls/d) } \\
\hline$<3$ & $26 \cdot 4$ & $24 \cdot 9$ & $1 \cdot 00$ & & & $1 \cdot 00$ & & \\
\hline$\geq 3$ & $73 \cdot 6$ & $18 \cdot 7$ & $0 \cdot 88$ & $0 \cdot 61,1 \cdot 27$ & 0.507 & $0 \cdot 85$ & $0 \cdot 58,1 \cdot 26$ & 0.425 \\
\hline \multicolumn{9}{|c|}{ Tea (times/week) } \\
\hline 0 & $48 \cdot 2$ & $22 \cdot 7$ & $1 \cdot 00$ & & & $1 \cdot 00$ & & \\
\hline 1 or $2 /$ week & $18 \cdot 4$ & $21 \cdot 5$ & $1 \cdot 19$ & $0 \cdot 78,1 \cdot 80$ & 0.416 & $1 \cdot 24$ & $0 \cdot 80,1 \cdot 93$ & $0 \cdot 342$ \\
\hline$\geq 3$ & $33 \cdot 4$ & $16 \cdot 3$ & $0 \cdot 80$ & $0 \cdot 55,1 \cdot 18$ & $0 \cdot 265$ & $0 \cdot 77$ & $0.51,1 \cdot 16$ & $0 \cdot 211$ \\
\hline
\end{tabular}

*The model excluded those who had depressive symptoms (CES-D $\geq 10$ ) at baseline and those who failed to complete or died before the 2003 survey. Demographic and food consumption frequencies were baseline (1999) data and all variables were endpoint (2003) data. The model was controlled for baseline age, gender and years of formal education, satisfaction with economic status, living setting, smoking status, alcohol drinking, betel-nut chewing, functional status, physical activity, cognitive status (SPMSQ score) and the presence of major chronic co-morbidities (hypertension, diabetes, heart disease, cancer, stroke, chronic kidney disease, gout, joint pain/arthritis, gallbladder/liver disease, hip fracture and lower-back pain) at endpoint. All values were weighting-adjusted according to study design.

an increased risk of depressive disorders; Konttinen et al. ${ }^{(23)}$ observed that lower consumption of fruits and vegetables was related to higher depressive symptoms in 25-64-yearold Finnish men and women; and Oishi et al. ${ }^{(24)}$ observed that high carbohydrate intake was inversely associated with depression. In addition to dietary components, healthier dietary patterns have also been observed to be associated with reduced risks of depression. Nanri et al. ${ }^{(7)}$ observed that a healthy Japanese dietary pattern characterized by high intakes of fruits, vegetables, mushrooms and soya products was associated with fewer depressive symptoms in Japanese municipal employees. Kuczmarski et $a l^{(5)}$ observed that good diet quality (as indicated by the Healthy Eating Index) was significantly associated with reported symptoms of depression in African-American and white adults (30-64 years old) in the Baltimore area. Jacka et al. ${ }^{(6,25)}$ observed that a 'traditional' dietary pattern characterized by vegetables, fruits, meat, fish and whole grains was associated with lower odds for major depression or dysthymia and for anxiety disorders, whereas a 'Western' diet of processed or fried foods, refined grains, sugary products and beer was associated with higher psychological symptoms in adults. A Mediterranean dietary pattern with adequate intakes of fruits, nuts, vegetables, cereals, legumes and fish, important sources of nutrients, has been linked to a lower risk of depression ${ }^{(26)}$.

There are only a few longitudinal studies that document the depression-protective effect of diet. Akbaraly et $a l^{(8)}$ observed that a processed foods dietary pattern (heavily loaded with sweetened desserts, fried food, processed meat, refined grains and high-fat dairy products) was a risk factor for depression five years later whereas a whole foods pattern (heavily loaded with vegetables, fruits and fish) was protective in middle-age persons $\left(55 \cdot 7\right.$ (SD 6) years). Sanchez-Villegas et $a l^{(27)}$ observed that a Mediterranean dietary pattern high in vegetables, fruits and nuts, cereal, legumes and fish and moderation in alcohol was linked to depression prevention in college graduates. These studies suggest a possible causal relationship between dietary patterns and 
the risk of depression but cannot pinpoint the food groups responsible for the effect. The present study has specifically identified a food group, vegetables, to be associated with reduced risk of depression four years later in older adults.

Although fruits and vegetables have similarities in chemical composition, these two food groups exhibited somewhat different degrees of association with the risk of depression in the present study. Both food groups were significantly associated with reduced risk of depression in a regression model without being adjusted for cognitive status. Adjustment of the regression model for cognitive status attenuated the association of depression with fruit consumption but not with vegetable consumption. This suggests that the association with fruits is mediated through cognitive status and fruit consumption may impact cognitive status. The mechanism of this relationship warrants further investigation.

In addition to vegetables and fruits, the present results also suggested that eggs may offer protection against depressive symptoms in older adults. Egg consumption was close to being statistically significant in both regression models $(P=0.087$ without and 0.069 with adjustment for cognitive status). Eggs are a good source of high-quality amino acids and rich in vitamins, minerals, $\alpha$-linolenic acid (an $n$-3 fatty acid) and natural antioxidants. Most of these food components have been implicated to play a role in reducing the risk of depression ${ }^{(10,11)}$.

\section{Potential mechanism}

The mechanism by which vegetables or 'healthy dietary patterns' reduces the risk of depression is not clearly known. Several dietary components such as the B-vitamins (especially $\mathrm{B}_{6}, \mathrm{~B}_{12}$ and folate which are involved in the metabolism of homocysteine), $n-3$ fatty acids and antioxidants have often been implicated to play a role. The intakes and plasma levels of $\mathrm{B}_{6}, \mathrm{~B}_{12}$ and folate have been found to be associated with depressive symptoms ${ }^{(10,11)}$ and supplementation with these vitamins has been shown to be effective in reducing the risk of symptoms. A prospective study has observed an association of vitamin $\mathrm{B}_{6}$, vitamin $\mathrm{B}_{12}$ and folate with depressive symptoms among older adults over time ${ }^{(28)}$. People who consume vegetarian diets are associated with healthy mood states. Lucas et al. ${ }^{(29)}$ observed that higher $\alpha$-linolenic acid and lower linoleic acid (an $n-6$ fatty acid) intakes reduce depression risk in a 10-year prospective study. Non-nutrient phytochemicals in fruits and vegetables could also play a role. These compounds have antioxidant properties. However, supplementation of $\alpha$-tocopherol (a major antioxidant nutrient) did not reduce depressive status in the elderly although plasma vitamin $\mathrm{E}$ was lower in persons with depression ${ }^{(30,31)}$.

It should also be mentioned that although the frequency of intake of vegetables (and maybe fruits and eggs) can predict future risk of depression in longitudinal studies, the possibility of reverse causality still cannot be totally excluded because the relationship between food and mood is bidirectional and both food pattern and depressive symptoms can be affected by events in daily life or the living environment ${ }^{(32)}$. Additionally, dietary patterns suggest nutrient intakes but also reflect personality or mood, which is a variable that cannot be easily controlled in regression models. Therefore, there is always a possibility that the observed effect is at least partially due to personality/mood or the way foods are prepared or consumed ${ }^{(33,34)}$.

\section{Strength and limitations}

The primary strength of our study is that the observed associations are based on longitudinal data from a large, population-based study with validated dietary assessment. Study results should have good generalizability. The study analysed dietary groups instead of dietary patterns, making it possible to identify specific food groups associated with risk of depression. However, the study also has some limitations. First are differences in food groupings among studies that may make comparisons difficult. Second, the FFQ is a well-established method but is not without its shortcomings. Quantification of food intake is difficult, especially in the elderly, and the data set contained frequency but without quantity (such as servings or portion size), thus quantifying food intake was not possible. Third, the study data were self-reported and have the usual limitations; self-reports generally have acceptable accuracy but some shortcomings are unavoidable.

\section{Conclusions}

Results suggest that what we eat can influence our psychological health. More frequent consumption of vegetables appears protective against depressive symptoms over time in older persons. These results have practical implications in geriatric health promotion. Older people should be encouraged to consume more vegetables since ageing is associated with a decrease in vegetable consumption. However, confounding factors could not be totally ruled out and the biochemical mechanism involved is largely unknown. Further research, especially well-controlled interventional studies to help clarify the role of foods and nutrients on depression, is warranted.

\section{Acknowledgements}

The study was supported by a grant from the National Science Council of Taiwan (NSC 97-2320-B-468-003). None of the authors have any conflict of interest. A.C.T. conceived the idea, directed the study and drafted the manuscript. T.-L.C. and S.-H.C. performed statistical analyses and helped revise the manuscript. The authors wish to express their appreciation to the Bureau of Health Promotion of the Department of Health of Taiwan for providing the data sets for this analysis. 


\section{References}

1. Williamson C (2009) Dietary factors and depression in older people. Br J Community Nurs 14, 422-426.

2. Freeman MP (2010) Nutrition and psychiatry. Am J Psychiatry 167, 244-247.

3. Woo J, Lynn H, Lau WY et al. (2006) Nutrient intake and psychological health in an elderly Chinese population. Int J Geriatr Psychiatry 21, 1036-1043.

4. Mikolajczyk R, El Ansari W \& Maxwell AE (2009) Food consumption frequency and perceived stress and depressive symptoms among students in three European countries. Nutr J 8, 31 .

5. Kuczmarski MR, Sees AC, Hotchkiss L et al. (2010) Higher Healthy Eating Index-2005 scores associated with reduced symptoms of depression in an urban population: findings from the Healthy Aging in Neighborhoods of Diversity Across the Life Span (HANDLS) Study. J Am Diet Assoc 110, 383-389.

6. Jacka FN, Pasco JA, Mykletun A et al. (2010) Association of Western and traditional diets with depression and anxiety in women. Am J Psychiatry 167, 305-311.

7. Nanri A, Kimura Y, Matsushita Y et al. (2010) Dietary patterns and depressive symptoms among Japanese men and women. Eur J Clin Nutr 64, 832-839.

8. Akbaraly TN, Brunner EJ, Ferrie JE et al. (2009) Dietary pattern and depressive symptoms in middle age. $\mathrm{Br} J$ Psychiatry 195, 408-413.

9. Wang JJ (2001) Prevalence and correlates of depressive symptoms in the elderly of rural communities in Southern Taiwan. J Nurs Res 9, 1-12.

10. Kim JM, Stewart R, Kim SW et al. (2008) Predictive value of folate, vitamin $\mathrm{B}_{12}$ and homocysteine levels in late-life depression. Br J Psychiatry 192, 268-274.

11. Skarupski KA, Tangney C, Li H et al. (2010) Longitudinal association of vitamin B-6, folate and vitamin B-12 with depressive symptoms among older adults over time. $\mathrm{Am} \mathrm{J}$ Clin Nutr 92, 330-335.

12. Bureau of Health Promotion, Department of Health, Taiwan (2007) 1989 Survey of the Elderly in Taiwan. http://www. bhp.doh.gov.tw/BHPnet/Portal/Them_Show.aspx?Subject= $200712270002 \&$ Class $=2 \& N o=200712270015$ (accessed July 2011).

13. Tsai AC \& Chang TL (2010) The effectiveness of BMI, calf circumference and mid-arm circumference in predicting subsequent mortality risk in elderly Taiwanese. $\mathrm{Br} \mathrm{J} \mathrm{Nutr}$ 105, 275-281.

14. Wu SC, Li CY \& Ke DS (2000) The agreement between selfreporting and clinical diagnosis for selected medical conditions among the elderly in Taiwan. Public Health 114, 137-142.

15. Goldman N, Lin IF, Weinstein M et al. (2003) Evaluating the quality of self-reports of hypertension and diabetes. J Clin Epidemiol 56, 148-154.

16. Boey KW (1999) Cross-validation of a short form of the CES-D in Chinese elderly. Int J Geriatr Psychiatry 14, 608-617.

17. Ofstedal MB, Zimmer ZS \& Lin HS (1999) A comparison of correlates of cognitive functioning in older persons in
Taiwan and the United States. J Gerontol B Psychol Sci Soc Sci 54, issue 5, S291-S301.

18. Pfeiffer E (1975) A short portable mental status questionnaire for the assessment of organic brain deficit in elderly patients. J Am Geriatr Soc 23, 433-441.

19. Fitti JE \& Kovar MG (1987) The supplement on aging to the 1984 National Health Interview Survey. Vital Health Stat 1 21, $1-115$.

20. Johnson JK, Lui LY \& Yaffe K (2007) Executive function, more than global cognition, predicts functional decline and mortality in elderly women. J Gerontol A Biol Sci Med Sci 62, 1134-1141.

21. Avila-Funes A, Garant MP \& Aguilar-Navarro S (2006) Relationship between determining factors for depressive symptoms and for dietary habits in older adults in Mexico. Pan Am J Public Health 19, 321-330.

22. Verger P, Lions C \& Ventelow B (2009) Is depression associated with health risk-related behavior clusters in adults? Eur J Public Health 19, 618-624.

23. Konttinen $\mathrm{H}$, Mannisto S, Sarlio-Lahteenkorva $\mathrm{S}$ et al. (2010) Emotional eating, depressive symptoms and selfreported food consumption. A population-based study. Appetite 54, 473-479.

24. Oishi J, Doi H \& Kawakami N (2009) Nutrition and depressive symptoms in community-dwelling elderly persons in Japan. Acta Med Okayama 63, 9-17.

25. Jacka FN, Mykletun A, Berk M et al. (2011) The association between habitual diet quality and the common mental disorders in community-dwelling adults: the Hordaland Health study. Psychosom Med 73, 483-490.

26. Sanchez-Villegas A, Henriquez $\mathrm{P}$, Bes-Rastrollo $\mathrm{M}$ et al. (2006) Mediterranean diet and depression. Public Health Nutr 9, 1104-1109.

27. Sanchez-Villegas A, Delgado-Rodriguez M \& Alonso A (2009) Association of the Mediterranean dietary pattern with the incidence of depression. Arch Gen Psychiatry 66, 1090-1098.

28. Merete C, Falcon LM \& Tucker KL (2008) Vitamin $B_{6}$ is associated with depression symptomatology in Massachusetts elders. J Am Col Nutr 27, 421-427.

29. Lucas ML, Mirzaei M, O'Reilly EJ et al. (2011) Dietary intake of $n-3$ and $n-6$ fatty acids and the risk of depression in women: a 10-y prospective follow-up study. Am J Clin Nutr 93, 1337-1343.

30. Tiemeier H, Hofman A, Kiliaan AJ et al. (2002) Vitamin E and depressive symptoms are not related. The Rotterdam Study. J Affect Disord 72, 79-83.

31. Owen AJ, Batterham MJ, Probst YC et al. (2005) Low plasma vitamin E levels in major depression: diet or disease? Eur J Clin Nutr 59, 304-306.

32. Christensen L \& Pettijohn L (2001) Mood and carbohydrate cravings. Appetite 36, 137-145.

33. Gibson EL (2006) Emotional influences on food choice: sensory, physiological and psychological pathways. Physiol Behav 89, 53-61.

34. Kimura Y, Wada T, Ishine M et al. (2009) Food diversity is closely associated with activities of daily living, depression, and quality of life in community-dwelling elderly people. J Am Geriatr Soc 57, 922-924. 